Document downloaded from:

http://hdl.handle.net/10251/163200

This paper must be cited as:

Palma, R.; Pérez-Aparicio, JL.; Taylor, RL. (2020). Non-linear and hysteretical finite element formulation applied to magnetostrictive materials. Computational Mechanics. 65(6):14331445. https://doi.org/10.1007/s00466-020-01828-y

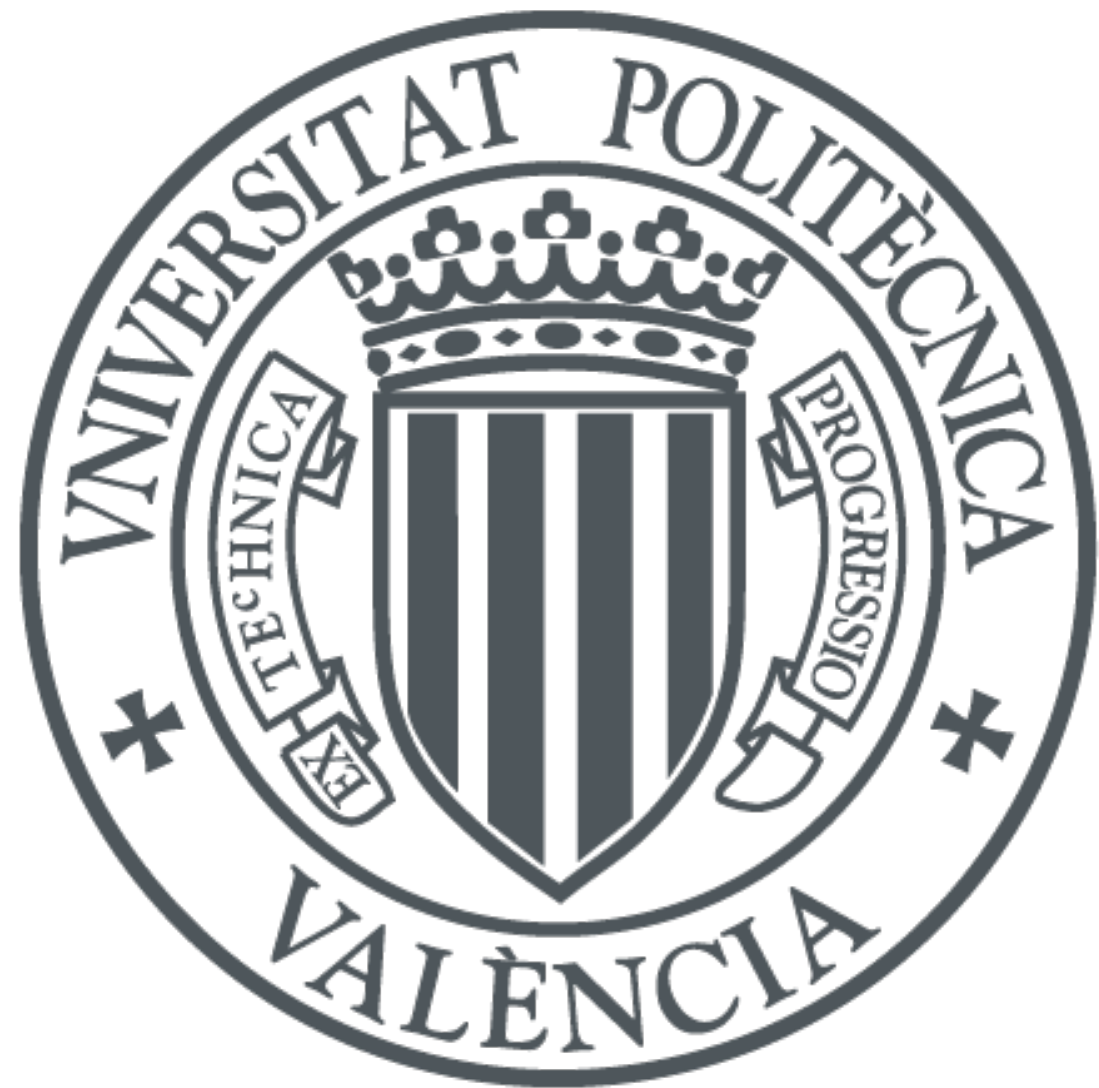

The final publication is available at

https://doi.org/10.1007/s00466-020-01828-y

Copyright Springer-Verlag

Additional Information

The final publication is available at link.springer.com. 


\title{
Non-linear and hysteretical finite element formulation applied to magnetosctrictive materials
}

\author{
Roberto Palma • José L. Pérez-Aparicio • Robert L. Taylor
}

Received: date / Accepted: date

\begin{abstract}
Giant magnetostrictive actuators are suitable for applications requiring large mechanical displacements under low magnetic fields; for instance Terfenol$\mathrm{D}$ made out of rare earth-iron materials can produce important strains. But these actuators exhibit hysteretic non-linear behavior, making it very difficult to experimentally characterize them. Therefore, sophisticated numerical algorithms to develop computational tools are necessary. In this work, theoretical and numerical formulations within the finite element method are developed to simulate magnetostriction. Theoretically, within the framework of non-equilibrium thermodynamics, the hysteresis is introduced by the Debye-memory relaxation. Numerically, the main novelty is the time integration, coupled Newmark- $\beta$ (for mechanical) and convolution integrals (for magnetic constitutive equations); the non-linearity is solved with the standard Newton-Raphson algorithm. Constitutive non-linearities are incorporated with the Maxwell stress tensor, quadratically dependent on the magnetic field. The numerical code is validated using analytical and experimental solutions; several examples are presented to demonstrate the capabilities of the present formulation.
\end{abstract}

Roberto Palma

Department of Mechanical Engineering and Construction, Universitat Jaume I, Castellón de la Plana, Spain

E-mail: rpalma@uji.es

José L. Pérez-Aparicio

Department of Continuum Mechanics and Theory of Structures, Universitat Politècnica de València, Valencia, Spain

Robert L. Taylor

Department of Civil and Environmental Engineering, University of California at Berkeley, California, USA
Keywords Finite Element Method · Magnetostrictive - Maxwell stress tensor · Magnetic Debye memory · Convolution Integrals $\cdot$ Hysteresis

\section{Introduction}

Nowadays, sensors and actuators are typically used in many mechatronic devices, for instance those assembled in smart structures for civil and aeronautic engineering [1], medical applications [2], energy harvesting [3] and robotics [4]. For this reason the study, understanding and characterization of smart materials are important challenges for the theoretical, experimental and numerical scientific and engineering communities.

Smart materials such as piezoceramics are common in applications that require small mechanical displacements. On the contrary, rare earth-iron materials exhibiting giant magnetostriction under relatively low applied magnetic fields, are more suitable for large-displacement applications. However, the main drawback of these magnetostrictive materials emerges from their inherent magnetism resulting in hysteretic non-linearities. In particular, this hysteresis produces double-valued responses and the accurate experimental characterization of magnetostrictive materials becomes very difficult if not impossible. Therefore, a robust numerical formulation of magnetostrictive materials, including non-linearities and hysteresis phenomena, is of central importance to develop computational tools for the proper characterization of these materials by using, for example, inverse problems based on models.

In the literature, there is a wide range of experimental and theoretical works on magnetostrictive actuators $[5,6,7]$. Numerically, the electrical engineering community has developed several formulations based 
on equivalent physical models, for example see [8]. In contrast, the continuum mechanics community often uses the Finite Element (FE) method to model magnetostriction. In this sense, non-linear FE formulations based either on vector potential [9] — requiring to model both the ferromagnetic part and the coil-, or scalar potential [10] - facilitating the application of boundary conditions but missing some physics - have been developed by the authors of the present work.

In the framework of the FE method, non-linearities are introduced either by: i) the consideration of the Maxwell stress tensor (MST) in the formulation, as in $[9,10,11]$ or, ii) the introduction of non-linear constitutive equations [12]. The first procedure is more robust than the second since it does not require complex trial and error calibrations that depend on the material. In addition, the MST is the best physical descriptor of non-linearities, as argued in [13].

Concerning the hysteresis modeling, there exist several approaches in the literature:

- Phenomenological-based approaches as in [14], the main drawback of which is an additional lack of robustness.

- Models with hysteresis operators such as Preisach and Jiles-Atherton, for instance $[8,15]$.

- Thermodynamic consistent models, based on macroscopic descriptions that satisfy the second law of thermodynamics. The independent variables of the internal energy are split into reversible and irreversible contributions, [16].

The present work adheres to the last approach and presents a formulation and a variational development based on FE to numerically study the non-linear behavior of magnetostrictive actuators.

Theoretically, non-linearities are introduced in the linear momentum balance by the MST, while the hysteresis of the magnetic field is incorporated with an irreversible term in the total internal energy, according to non-equilibrium thermodynamics [17]. The presence of this term results in a frequency-dependence governed by a relaxation time, producing a magnetic viscositylike behavior.

Numerically, a displacement-based weak formulation, which considers scalar magnetic potential is developed. The weak forms are discretized by standard three-dimensional shape functions of the Lagrangian type, and a monolithic residual-based formulation is developed to solve the non-linearities by the standard Newton-Raphson algorithm. One of the main novelties of the present work is the time integration scheme that combines the Newmark- $\beta$ algorithm for mechanical dynamics [18] with convolution integrals for the time-dependent constitutive equations.
The numerical formulation is implemented into the research code FEAP [20], from the University of California at Berkeley (USA). The numerical tool is validated against analytical and experimental results and, finally, two time-dependent electric intensities are prescribed in the model of a commercial magnetostrictive actuator to show the benefits of the present formulation.

Several simplifications are assumed:

i) For the mechanic field, small strain and absence of dissipative effects such as viscoelasticity or plasticity.

ii) Since the scalar magnetic potential will be used, electric flux sources $\boldsymbol{j}^{f}$ are dropped. Assuming low electromagnetic frequencies, the electromagnetic momentum will be neglected in comparison to the mechanic one.

iii) Adiabatic processes: the effects of temperature $T$ are not considered.

iv) The constitutive tensors are considered constant, not function of the magnetic field or $T$.

The simplification i) is here a good approximation due to the relative high stiffness of the magnetostrictive material. In ii), the two assumptions imply that only ferromagnetic materials can be modeled, but in exchange the magnetic field generated by coils can be easily prescribed through the Biot-Savart's law, as in $[11,15]$. Assuming iv) means that the magnetostrictive material cannot be studied in full saturation: this is again not a shortcoming since Mini Actuators (MA) do not usually work in this zone.

Finally, Cartesian coordinates are assumed and the symbols $\left({ }^{\cdot}\right),\left({ }^{*}\right), \nabla, \mathbf{a} \cdot \mathbf{b}=\mathbf{a}^{\top} \mathbf{b}, \mathbf{a}: \mathbf{b}=\left(\mathbf{a} \cdot \mathbf{b}^{\top}\right): \boldsymbol{I}$, $\mathbf{a} \otimes \mathbf{b}=\mathbf{a} \mathbf{b}^{\top},()^{\top},(\cdot),{ }_{j}$ denote time derivative, double time derivative, del or nabla operator, dot product, double contraction product, outer product, transposition and differentiation with respect to the $j$-coordinate, respectively. In addition, $\boldsymbol{I}$ denotes the second-order identity tensor.

\section{Theoretical formulation}

The aim of this section is to develop the governing equations for the hysteretic non-linear magnetostrictive model. To this end, the linear and angular momentum balances are obtained from classical continuum mechanics theory and from Maxwell's laws of electromagnetism. Then, the compatibility equations are stated and a non-equilibrium thermodynamic approach is performed to obtain the constitutive equations. Finally, boundary and initial conditions are introduced. 


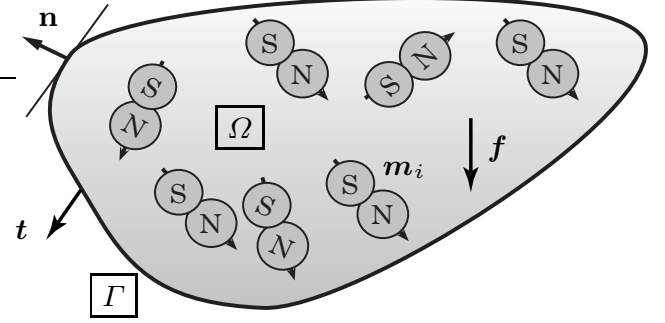

Fig. 1 A magnetostrictive domain $\Omega$, its boundary $\Gamma$ with outward normal $\mathbf{n}$; randomly oriented magnetic dipoles $\boldsymbol{m}_{i}$, body forces $f$ and boundary tractions $t$.

\subsection{Momentum balances}

Consider the generic solid of Fig. 1; the magnetic nature of the medium has been represented by a number $i$ of magnetic dipoles with north and south poles, and the magnetic moment of each dipole is denoted by $\boldsymbol{m}_{i}$.

Since $\Omega$ is filled with magnetostrictive material, the momentum balances must include mechanic and electromagnetic contributions. The latter will be obtained from the magnetic Maxwell's laws [22] in the absence of $\boldsymbol{j}^{f}$ :

$$
\nabla \cdot \boldsymbol{B}=0, \quad \nabla \times \boldsymbol{H}-\frac{\partial \boldsymbol{D}}{\partial t}=\boldsymbol{O},
$$

where $\boldsymbol{D}, \boldsymbol{B}$ and $\boldsymbol{H}$ denote electric displacement, magnetic induction and magnetic field, respectively. Since in the present model there are no electric degrees of freedom, the electric Maxwell equations do not need to be included.

Associated to these laws, the electromagnetic constitutive equations that relate magnetizations $\boldsymbol{M}$ with both $\boldsymbol{H}$ and $\boldsymbol{B}$ is expressed as:

$$
\boldsymbol{B}=\mu_{0}(\boldsymbol{H}+\boldsymbol{M})
$$

where $\mu_{0}$ is the permeability of vacuum.

Given that the Lagrangian function is independent of the reference system and of the angle of measurement [21] and according to continuum physics, the domain must satisfy linear and angular momentum balances.

From [22], the electromagnetic linear momentum reads:

$\boldsymbol{f}_{E M}=\nabla \cdot \boldsymbol{\sigma}_{M x}^{\top}-\frac{\partial \boldsymbol{p}_{E M}}{\partial t}$,

where $\boldsymbol{\sigma}_{M x}, \boldsymbol{f}_{E M}$ and $\boldsymbol{p}_{E M}$ denote the MST, electromagnetic body forces and electromagnetic momentum, respectively. As commented, electromagnetic frequencies are greater than mechanical one and, consequently, $\boldsymbol{p}_{E M}$ is neglected.

While $\boldsymbol{f}_{E M}$ represents long-range forces related with ponderomotive forces due to electromagnetic sources, the MST is closely related to short-range forces, responsible for magnetostrictive behavior [13]; this tensor must be incorporated in the constitutive equations as argued in $[24,25]$. The magnetic part of the MST may be explicitly expressed as:

$\boldsymbol{\sigma}_{M x}=\boldsymbol{B} \otimes \boldsymbol{H}-\frac{\boldsymbol{B} \cdot \boldsymbol{B}}{2 \mu_{0}} \boldsymbol{I}$.

The symmetric form of (4) reads:

$\boldsymbol{\sigma}_{M x}^{s y}=\frac{\mu_{0} \boldsymbol{B} \otimes \boldsymbol{H}+\mu_{0} \boldsymbol{H} \otimes \boldsymbol{B}-\boldsymbol{B} \cdot \boldsymbol{B} \boldsymbol{I}}{2 \mu_{0}}$,

Inside matter and for anisotropic magnetostrictive materials, $\boldsymbol{\sigma}_{M x}$ is non-symmetric and therefore the angular momentum will not be conserved directly. To ensure the conservation, the sum of electromagnetic and mechanic momentums must result in a symmetric total stress tensor $\sigma_{T}:=\sigma_{C l}+\sigma_{M x}$, where $\sigma_{C l}$ is a nonsymmetric Cauchy-like tensor and $\boldsymbol{\sigma}_{T}$ is related with a theoretical mechatronic vector $\boldsymbol{t}_{E M}=\boldsymbol{\sigma}_{T}^{\top} \cdot \mathbf{n}$, see [23]. Since this "traction" $\boldsymbol{t}_{E M}$ is associated with a tensor, it represents the short-range forces and combines both electromagnetic and mechanic tractions.

Taking advantage of the previous definition of $\boldsymbol{\sigma}_{T}$, the total (mechanic and magnetic) linear momentum balance becomes:

$\frac{\mathrm{d}}{\mathrm{d} t} \int_{\Omega} \rho_{m} \dot{\boldsymbol{u}} \mathrm{d} \Omega=\oint_{\Gamma} \boldsymbol{t}_{E M} \mathrm{~d} \Gamma+\int_{\Omega} \boldsymbol{f} \mathrm{d} \Omega$,

where $\boldsymbol{u}$ denotes the mechanic displacement field, $\rho_{m}$ the mass density and $\boldsymbol{f}$ the body forces. Applying the divergence theorem to the first term in the right-hand side of (6), the total linear momentum in local form becomes:

$\rho_{m} \ddot{\boldsymbol{u}}=\nabla \cdot \boldsymbol{\sigma}_{T}^{\top}+\boldsymbol{f}$.

Since by construction $\boldsymbol{\sigma}_{T}=\boldsymbol{\sigma}_{T}^{\top}$, the total angular momentum is conserved as stated by the Noether's theorem, [21]. According to [28], the skew-symmetric parts of $\boldsymbol{\sigma}_{C l}$ and $\boldsymbol{\sigma}_{M x}$ cancel each other, and the symmetric part of the first is simply the Cauchy stress tensor $\boldsymbol{\sigma}_{C}$; then the total stress is rewritten as:

$\boldsymbol{\sigma}_{T}=\boldsymbol{\sigma}_{C}+\boldsymbol{\sigma}_{M x}^{s y}$

The present formulation allows to calculate $\boldsymbol{\sigma}_{C}$ from standard thermodynamic approaches of the mechanic field (see Section 2.3), and $\boldsymbol{\sigma}_{T}$ by the addition of two classical tensors.

The theory of total stress tensor and related aspects are the subject of an ongoing work [23]. 


\subsection{Compatibility equations}

Following a standard continuum mechanic approach, the compatibility equation for the mechanic field is obtained from the displacement gradient:

$\boldsymbol{u} \otimes \nabla=\nabla^{s y} \boldsymbol{u}:=\boldsymbol{S}$,

where only the symmetric part of the tensor is considered and the small strain tensor is denoted by $\boldsymbol{S}$.

Again assuming low frequencies $\partial \boldsymbol{D} / \partial t=0$, the right (1) or Ampère's law defines a scalar magnetic potential $\varphi$, according to the Helmholtz's decomposition theorem:

$\nabla \times \boldsymbol{H}=\boldsymbol{O} \quad \Rightarrow \quad \boldsymbol{H}=-\nabla \varphi$.

Then the Ampère's law of (1) is directly enforced from this choice of $\varphi$. For more details on scalar and vector potentials, the reader is referred to [10].

\subsection{Material constitution}

According to the theory of magnetism [26], there are two main types of magnetic materials depending on their dipole moment orientation:

- Paramagnetic: randomly oriented dipoles, Fig. 2 left.

- Ferromagnetic, such as magnetostrictives: dipoles predominantly parallel to each other, Fig. 2 middle.

For both of them the dipoles tend to align more parallel upon application of $\boldsymbol{H}$ and the material becomes more ferromagnetic; consequently, the effect $\boldsymbol{M}$ is proportional to the cause $\boldsymbol{H}$.

In practice, magnetic materials are neither perfectly paramagnetic nor perfectly ferromagnetic. Therefore, a momentary delay appears (Fig. 2 right) when the magnetic dipoles return to their original orientations upon removal of $\boldsymbol{H}$, as long as the coercive field has not been reached. This delay is measured by the magnetic relaxation time $\tau_{M}$ and its consideration will require the addition of an irreversible term in the total internal energy. Notice that this delay is similar to that of electric dipoles $\tau_{P}$ in the Debye memory effect for dielectric materials, [19].

Thermodynamically, the first and second laws of thermodynamics must be satisfied, and their rate expressions may are:

$$
\begin{aligned}
& \frac{\mathrm{d}}{\mathrm{d} t}(\mathcal{U}+\mathcal{K})=\frac{\mathrm{d}}{\mathrm{d} t}\left(\mathcal{Q}^{r}+\mathcal{W}^{r}\right)+\frac{\mathrm{d}}{\mathrm{d} t}\left(\mathcal{Q}^{i}+\mathcal{W}^{i}\right), \\
& T \frac{\mathrm{d} \mathcal{S}}{\mathrm{d} t} \geq \frac{\mathrm{d}}{\mathrm{d} t}\left(\mathcal{Q}^{r}+\mathcal{Q}^{i}\right),
\end{aligned}
$$
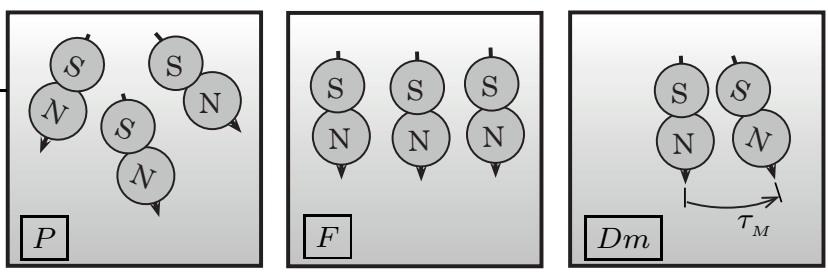

Fig. 2 Dipole moment orientation: paramagnetic $P$ (left), ferromagnetic $F$ (middle), magnetic Debye memory $D m$ (right). $\tau_{M}$ relaxation time for dipole to return to original position when magnetic field is removed.

where $\mathcal{U}, \mathcal{K}, \mathcal{Q}, \mathcal{W}$ and $\mathcal{S}$ denote internal energy, kinetic energy, heat, work and entropy, respectively. Since $\tau_{M}$ introduces dissipation, reversible $(\cdot)^{r}$ and irreversible magnitudes $(\cdot)^{i}$ have been defined and, consequently, the second law is described by the Clausius' inequality of the second equation of (11).

To expand (11), first the rate of internal and kinetic energies in $\Omega$ can be expressed in continuum form as:

$$
\frac{\mathrm{d}}{\mathrm{d} t}(\mathcal{U}+\mathcal{K})=\int_{\Omega} \rho_{m}(\dot{\mathrm{u}}+\ddot{\boldsymbol{u}} \cdot \dot{\boldsymbol{u}}) \mathrm{d} \Omega,
$$

where $\mathrm{u}$ denotes the specific internal energy.

Second, the rate of total entropy is also rewritten in continuum form. The reversible part is the entropy flux $\boldsymbol{j}_{\mathrm{s}}$ flowing through $\Gamma$ and the irreversible part is the entropy production $\Sigma_{\mathrm{s}}$ inside $\Omega$; mathematically:

$$
\frac{\mathrm{d} \mathcal{S}}{\mathrm{d} t}=-\oint_{\Gamma} \boldsymbol{j}_{\mathrm{s}} \cdot \mathbf{n} \mathrm{d} \Gamma+\int_{\Omega} \Sigma_{\mathrm{s}} \mathrm{d} \Omega
$$

Notice that the process would be reversible in absence of $\Sigma_{\mathbf{s}}$ and in this situation the second law given in (11) (bottom) becomes an equality.

Third, the rate of total work (including reversible and irreversible contributions) is given by:

$$
\begin{aligned}
\frac{\mathrm{d}}{\mathrm{d} t}\left(\mathcal{W}^{r}+\mathcal{W}^{i}\right) & =\oint_{\Gamma} \boldsymbol{t}_{E M} \cdot \dot{\boldsymbol{u}} \mathrm{d} \Gamma+\int_{\Omega} \boldsymbol{f} \cdot \dot{\boldsymbol{u}} \mathrm{d} \Omega \\
& +\int_{\Omega} \boldsymbol{H}^{r} \cdot \dot{\boldsymbol{B}} \mathrm{d} \Omega+\dot{\mathcal{W}}^{i} .
\end{aligned}
$$

Finally, applying the divergence to the boundary integrals, introducing (12), (13) and (14) in (11) and taking into account the linear momentum balance (7), the balance of energy in local form becomes:

$\rho_{m} \dot{\mathrm{u}}=-T \nabla \cdot \boldsymbol{j}_{\mathrm{s}}+T \Sigma_{\mathrm{s}}+\boldsymbol{\sigma}_{T}^{\top}: \dot{\boldsymbol{S}}^{r}+\boldsymbol{H}^{r} \cdot \dot{\boldsymbol{B}}+\dot{\mathcal{W}}^{i}$.

Since the process is adiabatic, the entropy fluxfirst term of (15) right-hand side - is neglected and two conclusions are extracted:

- In the absence of entropy production (reversible process), the total internal energy is a function of strain and magnetic induction, namely $\mathcal{U}=\mathcal{U}\left(\boldsymbol{S}^{r}, \boldsymbol{B}\right)$. 
- The entropy production that introduces dissipations, with:

is due to the irreversible rate of work:

$$
\Sigma_{\mathrm{s}}=-\frac{\dot{\mathcal{W}}^{i}}{T}
$$

where $\mathcal{W}^{i}$ contains only magnetic contributions due to assumption i) from Section 1.

From the energy defined in the first conclusion, a conservative constitutive equation is developed in Section 2.3.1. To satisfy the result of the second conclusion, a dissipative term deduced from non-equilibrium thermodynamics is applied in Section 2.3.2.

\subsubsection{Equilibrium thermodynamics}

The choice of the scalar magnetic potential (10), permits an amenable displacement-based FE formulation with degrees-of-freedom $\boldsymbol{u}$ and $\varphi$. For that, a Legendre transformation must be applied to $\mathcal{U}\left(\boldsymbol{S}^{r}, \boldsymbol{B}\right)$ to obtain an expression for a thermodynamic potential $\Pi\left(\boldsymbol{S}^{r}, \boldsymbol{H}^{r}\right)$. In other words, it is intended to exchange $\boldsymbol{B}$ by $\boldsymbol{H}^{r}$ as the depending magnetic variable. Furthermore, a natural state for which a residual stress $\boldsymbol{\sigma}_{R}$ exists is considered, since many of the magnetostrictive devices are preloaded.

Mathematically and in a first and good approximation, $\Pi$ may be calculated by a Taylor series expansion in the neighborhood of its natural state, see [10], to give:

$$
\begin{aligned}
\Pi\left(\boldsymbol{S}, \boldsymbol{H}^{r}\right)= & \boldsymbol{\sigma}_{R}: \boldsymbol{S}^{r}-\boldsymbol{e}^{\varphi}: \boldsymbol{S}^{r} \cdot \boldsymbol{H}^{r}+ \\
& \frac{\boldsymbol{S}^{r}: \mathrm{C}: \boldsymbol{S}^{r}-\boldsymbol{H}^{r} \cdot \boldsymbol{\mu} \cdot \boldsymbol{H}^{r}}{2},
\end{aligned}
$$

where $\mathbf{C}, \boldsymbol{\mu}$ and $e^{\varphi}$ denote the fourth-order elastic, second-order permeability and third-order piezomagnetic tensors, respectively.

Since $\Pi$ is linear and non-dissipative, $\boldsymbol{\sigma}_{C}$ and $\boldsymbol{B}$ are directly obtained by applying the standard thermodynamic approaches:

$$
\begin{aligned}
& \boldsymbol{\sigma}_{C}=\frac{\partial \Pi}{\partial \boldsymbol{S}^{r}}=\mathbf{C}: \boldsymbol{S}^{r}-\left(\boldsymbol{e}^{\varphi}\right)^{\top} \cdot \boldsymbol{H}^{r}+\boldsymbol{\sigma}_{R}, \\
& \boldsymbol{B}=-\frac{\partial \Pi}{\partial \boldsymbol{H}^{r}}=\boldsymbol{e}^{\varphi}: \boldsymbol{S}^{r}+\boldsymbol{\mu} \cdot \boldsymbol{H}^{r} .
\end{aligned}
$$

For the sake of convenience, these equations and their inverse are expressed in compact form as:

$$
\begin{gathered}
\left\{\begin{array}{c}
\boldsymbol{\sigma}_{C}-\boldsymbol{\sigma}_{R} \\
\boldsymbol{B}
\end{array}\right\}=\left[\begin{array}{cc}
\mathbf{C} & -\left(\boldsymbol{e}^{\varphi}\right)^{\top} \\
\boldsymbol{e}^{\varphi} & \boldsymbol{\mu}
\end{array}\right]\left\{\begin{array}{c}
\boldsymbol{S}^{r} \\
\boldsymbol{H}^{r}
\end{array}\right\}, \\
\left\{\begin{array}{c}
\boldsymbol{S}^{r} \\
\boldsymbol{H}^{r}
\end{array}\right\}=\left[\begin{array}{cc}
\boldsymbol{A}_{11} & \boldsymbol{A}_{12} \\
-\boldsymbol{A}_{21} & \boldsymbol{A}_{22}
\end{array}\right]\left\{\begin{array}{c}
\boldsymbol{\sigma}_{C}-\boldsymbol{\sigma}_{R} \\
\boldsymbol{B}
\end{array}\right\},
\end{gathered}
$$

$$
\begin{aligned}
& \boldsymbol{A}_{11}=\left[\mathbf{1}+\mathbf{C}^{-1}\left(\boldsymbol{e}^{\varphi}\right)^{\top} \boldsymbol{\mu}^{-1} \boldsymbol{e}^{\varphi}\right]^{-1} \mathbf{C}^{-1}, \\
& \boldsymbol{A}_{12}=\left[\mathbf{1}+\mathbf{C}^{-1}\left(\boldsymbol{e}^{\varphi}\right)^{\top} \boldsymbol{\mu}^{-1} \boldsymbol{e}^{\varphi}\right]^{-1} \mathbf{C}^{-1}\left(\boldsymbol{e}^{\varphi}\right)^{\top} \boldsymbol{\mu}^{-1}, \\
& \boldsymbol{A}_{21}=\left[\boldsymbol{I}+\boldsymbol{\mu}^{-1} \boldsymbol{e}^{\varphi} \mathbf{C}^{-1}\left(\boldsymbol{e}^{\varphi}\right)^{\top}\right]^{-1} \boldsymbol{\mu}^{-1} \boldsymbol{e}^{\varphi} \mathbf{C}^{-1}, \\
& \boldsymbol{A}_{22}=\left[\boldsymbol{I}+\boldsymbol{\mu}^{-1} \boldsymbol{e}^{\varphi} \mathbf{C}^{-1}\left(\boldsymbol{e}^{\varphi}\right)^{\top}\right]^{-1} \boldsymbol{\mu}^{-1} .
\end{aligned}
$$

\subsubsection{Non-equilibrium thermodynamics}

In the framework of non-equilibrium thermodynamics [17], the entropy production of (16) is closely related to both the irreversible strain $\boldsymbol{S}^{i}=\boldsymbol{S}-\boldsymbol{S}^{r}$ and the irreversible magnetic field $\boldsymbol{H}^{i}=\boldsymbol{H}-\boldsymbol{H}^{r}$ :

$\Sigma_{\mathrm{s}}=\frac{\dot{\boldsymbol{\sigma}}_{C}:\left(\boldsymbol{S}-\boldsymbol{S}^{r}\right)}{T}+\frac{\dot{\boldsymbol{B}} \cdot\left(\boldsymbol{H}-\boldsymbol{H}^{r}\right)}{T}$,

where $\dot{\boldsymbol{\sigma}}_{C}$ and $\dot{\boldsymbol{B}}$ denote flux-like magnitudes called rate of stress and of magnetic induction, respectively.

The time-dependent constitutive equation may be obtained as recommended in [17]: in a first and good approximation $\dot{\boldsymbol{\sigma}}_{C}$ and $\dot{\boldsymbol{B}}$ are by linearly related with $\boldsymbol{S}^{i}$ and $\boldsymbol{H}^{i}$. Operating Eqs. (21) to (22):

$\left\{\begin{array}{c}\dot{\boldsymbol{\sigma}}_{C} \\ \dot{\boldsymbol{B}}\end{array}\right\}=\left[\begin{array}{cc}\boldsymbol{A}_{11}^{-1} / \tau_{\sigma} & \boldsymbol{O} \\ \boldsymbol{O} & \boldsymbol{A}_{22}^{-1} / \tau_{M}\end{array}\right]\left\{\begin{array}{c}\boldsymbol{S}-\boldsymbol{S}^{r} \\ \boldsymbol{H}-\boldsymbol{H}^{r}\end{array}\right\}$.

As observed in (23), two new phenomenological coefficients, commonly called relaxation times $\tau_{\sigma}$ and $\tau_{M}$, are incorporated to take into account the material dissipation. These coefficients are measured as function of the frequency from indirect experiment, and in particu$\operatorname{lar} \tau_{M}$ is closely related to the magnetic Debye memory, as drawn in Fig. 2.

The coupling coefficients of (23) representing physical interactions, and are commonly neglected for most magnetostrictive applications at macro-scale in the absence of mechanical dissipations.

Finally, introducing (20) into (23), the time-dependent constitutive equations become:

$$
\begin{aligned}
\tau_{\sigma} \dot{\boldsymbol{\sigma}}_{C}+\boldsymbol{\sigma}_{C} & =\mathbf{C}: \boldsymbol{S}-\left(\boldsymbol{e}^{\varphi}\right)^{\top} \cdot \boldsymbol{H}+\boldsymbol{\sigma}_{R} \\
\tau_{M} \dot{\boldsymbol{B}}+\boldsymbol{B} & =\boldsymbol{e}^{\varphi}: \boldsymbol{S}+\boldsymbol{\mu} \cdot \boldsymbol{H} .
\end{aligned}
$$

The present work assumes $\tau_{\sigma}=0$ due to assumption i) from Section 1. 


\subsubsection{Constitutive equations}

The set of two coupled, time-depend and non-linear constitutive equations are finally obtained by combining (24) and (8) to give:

$$
\begin{aligned}
\boldsymbol{\sigma}_{T} & =\mathbf{C}: \boldsymbol{S}-\left(\boldsymbol{e}^{\varphi}\right)^{\top} \cdot \boldsymbol{H}+\boldsymbol{\sigma}_{M x}^{s y}+\boldsymbol{\sigma}_{R}, \\
\boldsymbol{B}_{, \tau}+\boldsymbol{B} & =\boldsymbol{e}^{\varphi}: \boldsymbol{S}+\boldsymbol{\mu} \cdot \boldsymbol{H}:=\boldsymbol{G} .
\end{aligned}
$$

At this point, several observations are highlighted:

- The material non-linearity is incorporated by adding the symmetric part of the MST.

- The definition $\boldsymbol{B}_{, \tau}:=\partial \boldsymbol{B} / \partial \tau$ has been applied and also it has been non-dimensionalized by the change of variable $\tau=t / \tau_{M}$.

- $\boldsymbol{G}$ is introduced to simplify the subsequent notation.

- Classical piezomagnetic equations are recovered if the dissipation coefficient $\tau_{M}=0$, the symmetric MST and the residual stress are neglected.

\subsection{Boundary and initial conditions}

The set of two coupled partial differential equations first of (1) and (7) along with the constitutives (25) is completed defining proper boundary and initial conditions.

In this work, boundary conditions are composed of Dirichlet and Neumann type. Furthermore, both conditions must include mechanical and electrical terms since

\begin{tabular}{|c|c|}
\hline Dirichlet type & Neumann type \\
\hline $\boldsymbol{u}=\overline{\boldsymbol{u}}$ & $\boldsymbol{\sigma}_{T}^{\top} \cdot \mathbf{n}=\overline{\boldsymbol{t}}_{E M}$ \\
\hline$\varphi=\bar{\varphi}$ & $\boldsymbol{B} \cdot \mathbf{n}=0$ \\
\hline
\end{tabular}
the problem is coupled:

where $\overline{\boldsymbol{u}}, \bar{\varphi}, \overline{\boldsymbol{t}}_{E M}$ denote prescribed displacements, scalar magnetic potential and mechatronic vector all of them on $\Gamma$, respectively. Notice that:

$-\overline{\boldsymbol{t}}_{E M}$ must combine both mechanical and electrical contributions since they cannot be independently measured, [28].

$-\boldsymbol{B} \cdot \mathbf{n}$ is nil due to the apparent absence of magnetic monopoles in nature.

Regarding initial conditions, mechanical displacements, velocities and magnetic induction require initial values, assumed to be zero in the present work:

$\boldsymbol{u}(t=0)=\dot{\boldsymbol{u}}(t=0)=\dot{\boldsymbol{B}}(t=0)=\boldsymbol{O}$.

\section{Finite element formulation}

The governing equations reported in the previous section are discretized in the present one.

\subsection{Weak forms}

As is common in the FE framework, the governing equations are expressed in weak forms. To this end, the balance equations are multiplied by arbitrary test functions of the degrees of freedom: $\delta \boldsymbol{u}$ and $\delta \varphi$. The divergence theorem is then applied and natural (Neumann) boundary conditions are enforced: from (7) and the first (1):

$$
\begin{aligned}
& \int_{\Omega}\left[\delta \boldsymbol{u} \cdot\left(\boldsymbol{f}-\rho_{m} \ddot{\boldsymbol{u}}\right)-\delta \boldsymbol{S}: \boldsymbol{\sigma}_{T}^{\top}\right] \mathrm{d} \Omega+\oint_{\Gamma_{t}} \delta \boldsymbol{u} \cdot \overline{\boldsymbol{t}}_{E M} \mathrm{~d} \Gamma=0, \\
& \int_{\Omega} \delta \boldsymbol{H} \cdot \boldsymbol{B} \mathrm{d} \Omega=0
\end{aligned}
$$

where the notation $\delta \boldsymbol{S}=\nabla^{s y}(\delta \boldsymbol{u})$ and $\delta \boldsymbol{H}=-\nabla \delta \varphi$ has been introduced for clarity.

\subsection{Discretizations}

The continuum domain of Fig. 1 is discretized into $N$ three-dimensional (3D) eight-node brick elements of domain $\Omega_{e}$ and boundary $\Gamma_{e}$, satisfying $\Omega \approx \sum^{N} \Omega_{e}$. Furthermore, standard shape functions $\mathcal{N}$ of Lagrangian type are used to interpolate Cartesian coordinates, degrees of freedom and test functions:

$$
\begin{aligned}
& \boldsymbol{x} \approx \mathcal{N}_{a} \tilde{\boldsymbol{x}}_{a}, \quad \boldsymbol{u} \approx \mathcal{N}_{a} \tilde{\boldsymbol{u}}_{a} \\
& \dot{\boldsymbol{u}} \approx \mathcal{N}_{a} \tilde{\boldsymbol{u}}_{a}, \quad \ddot{\boldsymbol{u}} \approx \mathcal{N}_{a} \tilde{\ddot{\boldsymbol{u}}}_{a}, \quad \delta \boldsymbol{u} \approx \mathcal{N}_{a} \delta \tilde{\boldsymbol{u}}_{a}, \\
& \varphi \approx \mathcal{N}_{a} \tilde{\varphi}_{a}, \quad \delta \varphi \approx \mathcal{N}_{a} \delta \tilde{\varphi}_{a}
\end{aligned}
$$

where $\tilde{\boldsymbol{x}}_{a}, \tilde{\boldsymbol{u}}_{a}, \tilde{\varphi}_{a}$ denote a nodal value at the local node (numbered $a$ or $b$ ) belonging to the element $e$. In the previous equation, the Einstein summation convention is adopted. Using these expansions, the discrete forms of the compatibility equations (9) and (10) become:

$$
\begin{gathered}
\boldsymbol{S} \approx \nabla^{s y} \mathcal{N}_{a} \tilde{\boldsymbol{u}}_{a}:=\mathcal{B}_{a}^{s y} \tilde{\boldsymbol{u}}_{a}, \\
\boldsymbol{H} \approx-\nabla \mathcal{N}_{a} \tilde{\varphi}_{a}:=-\mathcal{B}_{a} \tilde{\varphi}_{a} .
\end{gathered}
$$

Using now Voigt's notation with indexes 1, 2, 3, 4, 5, 6 $\equiv 11,22,33,12,23,13$, the B-matrices (30) are explicitly given by:

$$
\mathcal{B}_{a}^{s y}=\left[\begin{array}{ccc}
\mathcal{N}_{a, 1} & 0 & 0 \\
0 & \mathcal{N}_{a, 2} & 0 \\
0 & 0 & \mathcal{N}_{a, 3} \\
\mathcal{N}_{a, 2} & \mathcal{N}_{a, 1} & 0 \\
0 & \mathcal{N}_{a, 3} & \mathcal{N}_{a, 2} \\
\mathcal{N}_{a, 3} & 0 & \mathcal{N}_{a, 1}
\end{array}\right], \quad \mathcal{B}_{a}=\left\{\begin{array}{c}
\mathcal{N}_{a, 1} \\
\mathcal{N}_{a, 2} \\
\mathcal{N}_{a, 3}
\end{array}\right\}
$$


As commented previously and due to the dissimilarity between mechanical and magnetic equations, they are discretized by two different approaches.

\subsubsection{Mechanical discretizations: Newmark- $\beta$ method}

The mechanical constitutive (25) is directly discretized using (30) to give:

$$
\boldsymbol{\sigma}_{T}^{n+1} \approx \mathbf{C} \mathcal{B}_{a}^{s y} \tilde{\boldsymbol{u}}_{a}^{n+1}+\left(\boldsymbol{e}^{\varphi}\right)^{\top} \boldsymbol{B}_{a} \tilde{\varphi}_{a}^{n+1}+\left.\boldsymbol{\sigma}_{M x}^{s y}\right|^{n+1}+\boldsymbol{\sigma}_{R}
$$

The supra-indices $n+1$ and $n$ represent the current and previous time steps, respectivelly.

Now the time discretization of the displacements is solved using the standard Newmark- $\beta$ method:

$$
\begin{aligned}
& \tilde{\boldsymbol{u}}_{a}^{n+1}=\tilde{\boldsymbol{u}}_{a}^{n}+\Delta t \tilde{\boldsymbol{u}}_{a}^{n}+\Delta t^{2}\left[\left(\frac{1}{2}-\beta_{N}\right) \tilde{\ddot{\boldsymbol{u}}}_{a}^{n}+\beta_{N} \tilde{\ddot{\boldsymbol{u}}}_{a}^{n+1}\right] \\
& \tilde{\tilde{\boldsymbol{u}}}_{a}^{n+1}=\tilde{\dot{\boldsymbol{u}}}_{a}^{n}+\Delta t\left[\left(1-\gamma_{N}\right) \tilde{\ddot{\boldsymbol{u}}}_{a}^{n}+\gamma_{N} \tilde{\ddot{\boldsymbol{u}}}_{a}^{n+1}\right]
\end{aligned}
$$

where $\beta_{N}, \gamma_{N}$ are the parameters that control stability and accuracy and the time increment is $\Delta t=t_{n+1}-t_{n}$.

\subsubsection{Magnetic discretizations: Convolution integrals}

The magnetic constitutive (25) bottom is an ordinary differential equation that can be solved by convolution integrals. A preliminary attempt to numerically discretize this equation for electric Debye memory was developed by the authors of the present work in [19]. The present approach includes modifications for a better formulation.

By assumption iv) of Section 1 the properties are constant in the range of study; then applying the change of variable from (25) to the forward time $t_{n+1}=\tau_{M} \tau_{n+1}$, the second (25) is integrated using variation of parameters to give:

$\boldsymbol{B}(\tau)=\int_{0}^{\tau} \mathrm{e}^{-\left(\tau-\tau^{\prime}\right)} \boldsymbol{G}\left(\tau^{\prime}\right) \mathrm{d} \tau^{\prime}$

where $\tau^{\prime}$ is a generic adimensional time within the interval $\left[\tau_{n+1}, \tau_{n}\right]$. This equation has the same form as the one reported in [17] for Debye theory of dielectrics.
Evaluating at $\tau_{n+1}$ the previous integral and substituting the equality $\tau_{n+1}=\tau_{n}+\Delta \tau$ :

$$
\begin{aligned}
\boldsymbol{B}\left(\tau_{n+1}\right)= & {\left[\int_{0}^{\tau_{n}} \mathrm{e}^{-\left(\tau_{n+1}-\tau^{\prime}\right)} \boldsymbol{G}\left(\tau^{\prime}\right) \mathrm{d} \tau^{\prime}+\right.} \\
& \left.\int_{\tau_{n}}^{\tau_{n+1}} \mathrm{e}^{-\left(\tau_{n+1}-\tau^{\prime}\right)} \boldsymbol{G}\left(\tau^{\prime}\right) \mathrm{d} \tau^{\prime}\right]= \\
& \mathrm{e}^{-\Delta \tau} \boldsymbol{B}\left(\tau_{n}\right)+\int_{\tau_{n}}^{\tau_{n+1}} \mathrm{e}^{-\left(\tau_{n+1}-\tau^{\prime}\right)} \boldsymbol{G}\left(\tau^{\prime}\right) \mathrm{d} \tau^{\prime}
\end{aligned}
$$

Introducing an additional change of variable $\eta=\tau^{\prime}-\tau_{n}$, the tensor $\boldsymbol{G}\left(\tau^{\prime}\right)$ can be linearly interpolated between its value at $\tau_{n}$ (noted by $\boldsymbol{G}_{n}$ ) and that of $\tau_{n+1}$ (by $\left.\boldsymbol{G}_{n+1}\right)$ with:

$$
\boldsymbol{G}\left(\tau^{\prime}\right)=\boldsymbol{G}_{n}+\frac{\eta}{\Delta \tau}\left(\boldsymbol{G}_{n+1}-\boldsymbol{G}_{n}\right)
$$

Finally introducing (36) in the last (35) gives:

$$
\begin{aligned}
\boldsymbol{B}\left(\tau_{n+1}\right) & =\mathrm{e}^{-\Delta \tau} \boldsymbol{B}\left(\tau_{n}\right)+ \\
& \mathrm{e}^{-\Delta \tau} \int_{0}^{\Delta \tau} \mathrm{e}^{\eta}\left[\boldsymbol{G}_{n}+\frac{\eta}{\Delta \tau}\left(\boldsymbol{G}_{n+1}-\boldsymbol{G}_{n}\right)\right] \mathrm{d} \eta
\end{aligned}
$$

The previous integrals may now be evaluated in closed form as:

$$
\begin{aligned}
& \int_{0}^{\Delta \tau} \mathrm{e}^{\eta} \mathrm{d} \eta \quad=\mathrm{e}^{\Delta \tau}-1 \\
& \frac{1}{\Delta \tau} \int_{0}^{\Delta \tau} \eta \mathrm{e}^{\eta} \mathrm{d} \eta=\mathrm{e}^{\Delta \tau}-\frac{\mathrm{e}^{\Delta \tau}-1}{\Delta \tau} .
\end{aligned}
$$

With the definition $\phi(\Delta \tau)=\left(1-\mathrm{e}^{\Delta \tau}\right) / \Delta \tau$, the previous closed form results allow (37) to be written as the direct expression:

$$
\begin{aligned}
\boldsymbol{B}\left(\tau_{n+1}\right) & =\mathrm{e}^{-\Delta \tau} \boldsymbol{B}\left(\tau_{n}\right)+ \\
& \left(1-\mathrm{e}^{-\Delta \tau}\right) \boldsymbol{G}_{n}+[1-\phi(\Delta \tau)]\left(\boldsymbol{G}_{n+1}-\boldsymbol{G}_{n}\right)
\end{aligned}
$$

As observed, the function $\phi(\Delta \tau)$ is not defined at the origin; however, taking limits $\phi(0) \rightarrow 1$. Therefore, for small values of the time increment, this function is evaluated using a series expansion of the exponential. 


\subsection{Residuals and tangent matrices}

Since the present problem is non-linear, a residual-based FE formulation is grounded on mechanical and magnetic residuals $\mathcal{R}^{u}$ and $\mathcal{R}^{\varphi}$ minimized by the NewtonRaphson algorithm [29].

Mathematically, the residuals are calculated introducing the discretizations (29) and (30) into the weak forms (28):

$$
\begin{aligned}
\mathcal{R}_{a}^{u, n+1}= & -\int_{\Omega_{e}} \mathcal{B}_{a}^{s y \top} \boldsymbol{\sigma}_{T}^{n+1} \mathrm{~d} \Omega_{e}+\oint_{\Gamma_{e}} \mathcal{N}_{a}^{\top} \overline{\boldsymbol{t}} \mathrm{d} \Gamma_{e}+ \\
& \int_{\Omega_{e}} \mathcal{N}_{a}^{\top}\left(\boldsymbol{f}-\rho_{m} \mathcal{N}_{b} \tilde{\ddot{\boldsymbol{u}}}_{b}^{n+1}\right) \mathrm{d} \Omega_{e} \\
\mathcal{R}_{a}^{\varphi, n+1}= & \int_{\Omega_{e}} \mathcal{B}_{a}^{\top} \boldsymbol{B}^{n+1} \mathrm{~d} \Omega_{e} .
\end{aligned}
$$

There exist several procedures to numerically solve coupled problems. For instance, stagger techniques are based on two steps: first, magnetic distributions are obtained and then applied to the calculation of the mechanical displacements. These procedures require less calculation time but their results have lower accuracy than those obtained with monolithic approaches, for which fully coupled tangent stiffness matrices must be developed.

The present work uses a monolithic approach with the tangent matrices obtained from a linearization of the residuals with respect to the degrees of freedom $\tilde{\boldsymbol{u}}_{b}^{n+1}$ and $\tilde{\varphi}_{b}^{n+1}$. The resulting monolithic assembled system is:

$$
\left[\begin{array}{cc}
\mathcal{K}_{a b}^{u u}+c_{3} \mathcal{M}_{a b}^{u u} & \mathcal{K}_{a b}^{u \varphi} \\
\mathcal{K}_{a b}^{\varphi u} & \mathcal{K}_{a b}^{\varphi \varphi}
\end{array}\right]^{k}\left\{\begin{array}{l}
\mathrm{d} \tilde{\boldsymbol{u}}_{b}^{n+1} \\
\mathrm{~d} \tilde{\varphi}_{b}^{n+1}
\end{array}\right\}=\left\{\begin{array}{l}
\mathcal{R}_{a}^{u, n+1} \\
\mathcal{R}_{a}^{\varphi, n+1}
\end{array}\right\}
$$

where the tangent stiffness $\mathcal{K}$ and mass $\mathcal{M}$ matrices are explicitly reported in Section 7, and $k$ is the iteration number of the Newmark algorithm and the coefficient $c_{3}$ results from linearizing (33):

$$
\mathrm{d} \tilde{\boldsymbol{u}}_{a}^{n+1}=\beta_{N} \Delta t^{2} \mathrm{~d} \tilde{\ddot{\boldsymbol{u}}}_{a}^{n+1}, \quad c_{3}=\frac{1}{\beta_{N} \Delta t^{2}} .
$$

\section{Validations}

In order to validate the numerical implementation, this section presents three comparisons (called "cases") between numerical and analytical/experimental results.
In particular, a one-dimensional (1D) analytical solution is used for cases I and II, and case III compares 3D FE results against experimental responses reported in [5]. For clarity, all cases are summarized in Table 1.

The material $\mathrm{Tb}_{0.3} \mathrm{Dy}_{0.7} \mathrm{Fe}_{1.92}$ (with commercial name Terfenol-D) magnetized along its long dimension is considered for all cases. This material provides a giant magnetostriction of $1000-2000(\mathrm{ppm})$ at $50(\mathrm{kA} / \mathrm{m})$ according to [8], and its properties taken from [11] are listed in Table 2.

For cases I and II, a 1D fixed-free rod of length 6 $(\mathrm{mm})$ is studied. Magnetically, $\bar{\varphi}_{b}=0$ is prescribed at the fixed end and its value at the other end is given by $\bar{\varphi}_{t}=-N_{a} I_{a}$ (Biot-Savart law); in the previous expression $N_{a}$ and $I_{a}$ denote the number of turns in the coil and prescribed electric current, respectively.

The rod is free-to-expand, then $\boldsymbol{\sigma}_{T}$ is zero; from (25) and (5) a $1 \mathrm{D}$ closed solution for the strain $S_{33}$ (along the magnetization direction) versus the applied magnetic field $H_{3}$ reads:

$$
S_{33}=\underbrace{\frac{e_{33}^{\varphi}}{C_{33}^{H}} H_{3}}_{\text {linear }}+\underbrace{\frac{\left(\mu_{33}^{T}\right)^{2}-2 \mu_{0} \mu_{33}^{T}}{2 \mu_{0} C_{33}^{H}} H_{3}^{2}}_{\text {MST }}-\underbrace{\frac{\sigma_{R 33}}{C_{33}^{H}}}_{\text {Preload }}
$$

where $\mu_{33}^{T}=\mu_{33}^{S}+\left(e_{33}^{\varphi}\right)^{2} / C_{33}^{H}$ and $\sigma_{R 33}$ is a stress along the length from a compressive preload, applied prior to the magnetic field.

Figure 3 plots $S_{33}$ versus $H_{3}$ distribution calculated with FE for case I, considering and not considering the MST. The analytical and numerical solutions agree very well for both solutions, and the following points may be stated:

- w/o MST, the slope of the distribution is $d_{33}=$ $e_{33}^{\varphi} / C_{33}^{H}=4.3 \times 10^{-9}(\mathrm{~m} / \mathrm{A})$, the piezomagnetic coefficient provided by the manufacturer.

- w/ MST, the curve is slightly non-linear due to the $H_{3}^{2}$ dependency shown in the second term of (43).

Numerically, the last solution is reached after four iterations of the Newton-Raphson algorithm; in particular, a quadratic convergence is observed from the initial $7.17 \times 10^{2}$ to the final $6.17 \times 10^{-7}$ residual norm.

There is not much difference between the two distributions, but in a more realistic $3 \mathrm{D}$ case (see for instance that of Fig. 7) the effective coefficient $C_{33}^{H}$ in the denominators of (43) would be smaller due to the two Poisson effects and the quadratic MST term more important.

The influence of the residual stress (with values $\sigma_{R 33}=$ $10,50,100[\mathrm{MPa}])$ on the response of the magnetostrictive material, namely case II, is shown in Fig. 4. As observed, the residual stress shifts the curve and as expected, the strain decreases with the increase of $\sigma_{R 33}$; 


\begin{tabular}{|c|c|c|}
\hline Case & Purpose & Solution \\
\hline \hline I & 1D comparison with and without MST & Analytical \\
II & 1D comparison with MST and $\boldsymbol{\sigma}_{R}$ & $-"-$ \\
III & 3D comparison with MST and $\boldsymbol{\sigma}_{R}$ & Experimental \\
\hline
\end{tabular}

Table 1 Summary of cases for numerical validations: I and II compare numerical and one-dimensional analytical solutions; III numerical and experimental responses from [5].

\begin{tabular}{|c|c|c|}
\hline Property & Value & Units \\
\hline \hline$C_{11}^{H} / C_{33}^{H} / C_{44}^{H} / C_{66}^{H} / C_{12}^{H} / C_{13}^{H}$ & $116 / 162 / 89 / 86 / 77 / 78$ & $\times 10^{9}\left(\mathrm{~N} / \mathrm{m}^{2}\right)$ \\
& $580 / 700 / 550$ & $(\mathrm{~N} / \mathrm{A} \cdot \mathrm{m})$ \\
$e_{31}^{\varphi} / e_{33}^{\varphi} / e_{15}^{\varphi}$ & $8.9 / 10$ & $\times 10^{-6}(\mathrm{H} / \mathrm{m})$ \\
$\mu_{11}^{S} / \mu_{33}^{S}$ & 9250 & $\left(\mathrm{~kg} / \mathrm{m}^{3}\right)$ \\
$\rho_{m}$ &
\end{tabular}

Table 2 Material properties of Terfenol-D from [11]: $C_{i j}^{H}$ fourth-order stiffness tensor, $e_{i j}^{\varphi}$ third-order piezomagnetic tensor, $\mu_{i j}^{S}$ second-order permeability tensor and $\rho_{m}$ mass density. Supra-indices $H, \varphi$ and $S$ denote the constant magnitude at which the coefficient is measured.

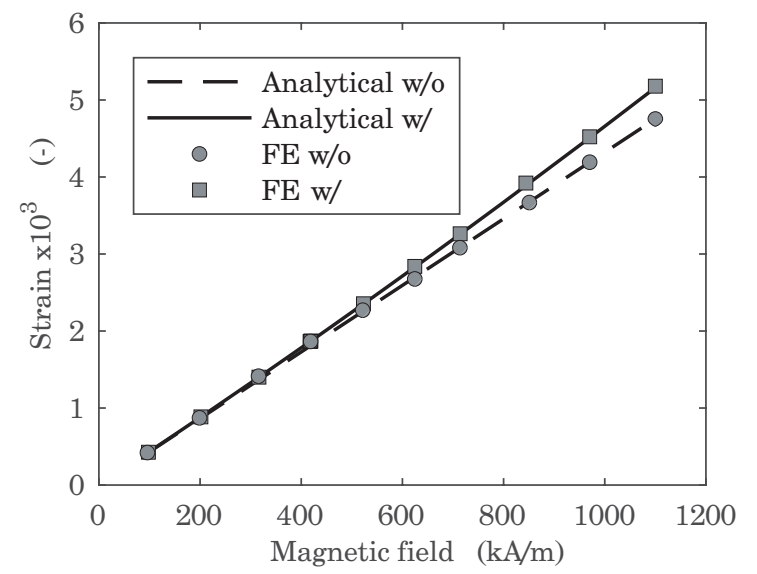

Fig. 3 Case I: Strain vs. applied magnetic field without (w/o) and with (w/) Maxwell stress tensor. Analytical (lines) and Finite Element (circles and rectangles).

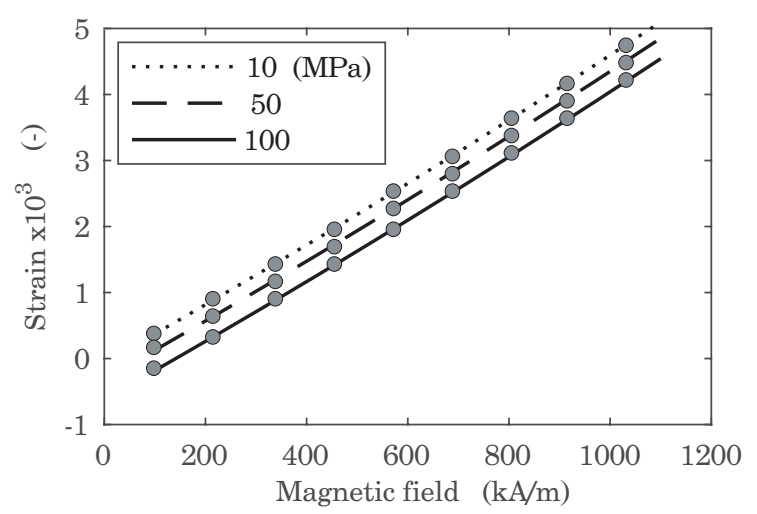

Fig. 4 Case II: Strain vs. applied magnetic for several residual stresses using Finite Elements and Maxwell tensor. Analytical (lines) and numerical (circles).

this reduction and the values fit very well with the experimental responses from [32].

In case III, the MA experimentally measured in [6] is modeled. The cross section of the device is shown in Fig. 5 and its geometric dimensions listed in Table

\begin{tabular}{|c|c|c|}
\hline Description & Value & Units \\
\hline \hline Rod length & 6 & $(\mathrm{~mm})$ \\
\hline Rod radius & 0.56 & $(\mathrm{~mm})$ \\
\hline End plate width & 3.86 & $(\mathrm{~mm})$ \\
\hline End plate thickness & 3.81 & $(\mathrm{~mm})$ \\
\hline Coil \# turns & 176 & - \\
\hline
\end{tabular}

Table 3 Geometrical dimensions of the magnetostrictive mini actuator from [5].

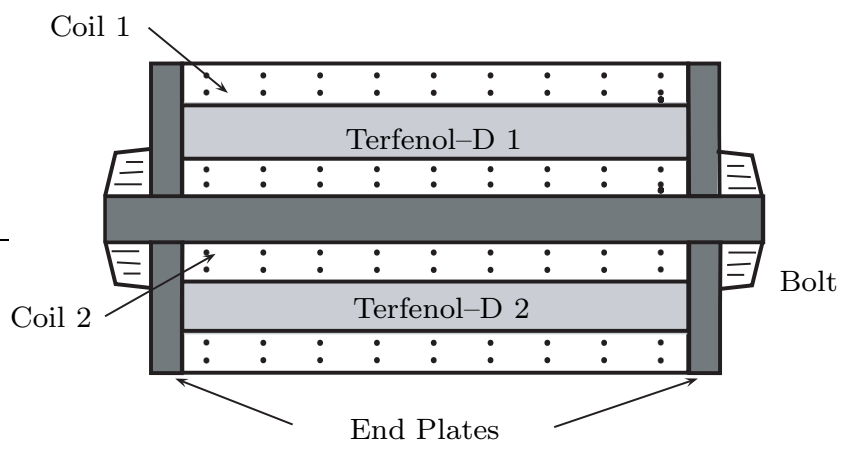

Fig. 5 Schematic representation of a magnetostrictive actuator (not cylindrical) cross section from [6]. Parts: active Terfenol-D rods, flat end plates, precompression bolt and magnetic coils.

3. The MA is composed of two cylindrical Terfenol-D rods, two flat end plates made out of Ni-Fe, two coils to generate the magnetic field and a bolt to preload the rods. According to [11], the magnetic permeability of the plates is three orders of magnitude greater than that of Terfenol-D, allowing for an easy closing of the magnetic lines.

The magnetic field along the rod axis is generated by the application of an electric current $I_{a}$ through the coils; due to the magneto-mechanical coupling, the end plates move. In this way, the electromagnetic field produces strain, due to the rotation of magnetic moments $\boldsymbol{m}_{i}$ as observed in Fig. 2 right. 


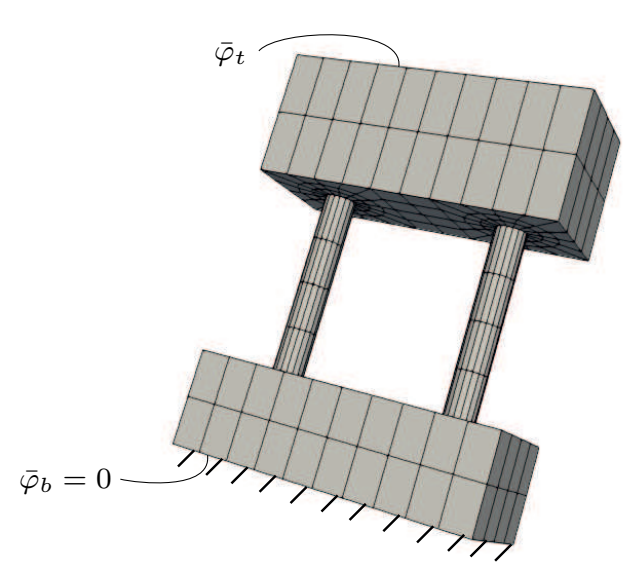

Fig. 6 Finite element mesh to simulate the MMA of Fig. 5: precompressed Terfenol-D rods and end iron plates.

From a modeling point of view, only the end plates and the Terfenol-D rods are discretized with a 3D mesh composed of 1,140 eight-noded elements as in Fig. 6. The coils do not need to be simulated since the magnetic field generated by them is calculated with BiotSavart's law.

The MA is mechanically clamped at the bottom plate, see Fig. 6 and as in the experiment of reference, the top end plate is free to move vertically as in the experiment. Magnetically, $\bar{\varphi}_{b}$ is set to zero at the bottom and the prescribed value at the top is again $\bar{\varphi}_{t}=-N_{a} I_{a}$, see Table 3 .

Figure 7 compares the experimental response reported in [5] (without the effect of the temperature) against the numerical results obtained by the present $\mathrm{FE}$ formulation, including the MST and under $\sigma_{R 33}=$ $9.6[\mathrm{MPa}]$ according to the reference.

If the MST term is not considered, the prediction is linear and only valid for small values of $I_{a}$ completely failing for medium or high values.

With MST, three different zones in the non-linear distribution may be observed:

$-I_{a}<100[\mathrm{~mA}]:$ the response is practically linear and both experimental and FE results fit very well.

$-100<I_{a}<320[\mathrm{~mA}]:$ the experimental response exhibits a strong concave shape that cannot exactly be captured by the FE results.

$-I_{a}>320[\mathrm{~mA}]$ : numerical and experimental results agree very well again.

The good agreement in the first and third zones could be due to the positions of the magnetic dipoles of Fig. 2: in the first and for a ferromagnetic magnetostrictive they are much aligned with $\boldsymbol{H}$ and in the second almost perpendicular (close to saturation) to $\boldsymbol{H}$; in these two zones, classical continuum mechanics is a good theory to model the response of the MA.

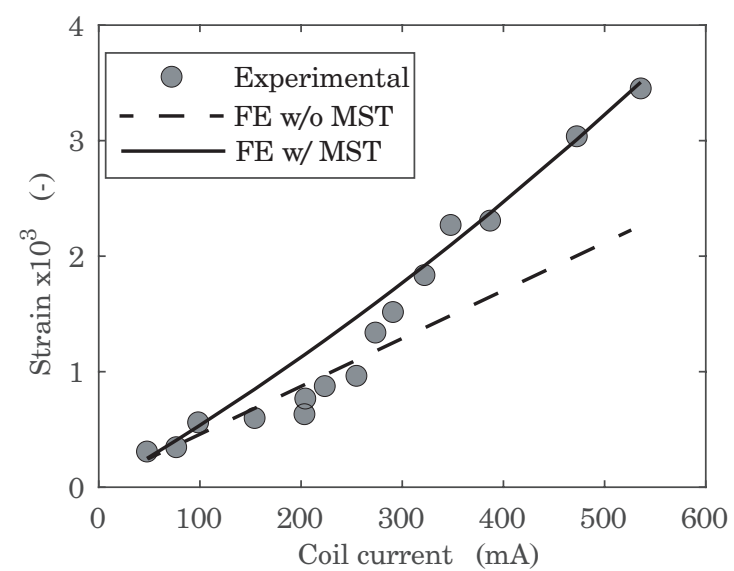

Fig. 7 Case III: Generated strain in the mini actuator vs. electric current applied to the coils; experimental (circles) from [6] and numerical (lines) results.

The disagreement of the second zone could be due to two reasons or the combination of both:

(a) Experimentally is, at least, difficult to measure the response due to the overheating of the coils, as argued in [5]: the influence of thermal strains has influence on the curve.

(b) Numerically, the present FE formulation has three main simplifications:

- As mentioned, the Joule's heating of the Terfenol$\mathrm{D}$ and of the coil is not incorporated in the model.

- The scalar potential FE formulation is a good approximation but assumes uniform $\boldsymbol{H}$ in the rods: in reality this field is not uniform due border effects.

- According to classical continuum mechanics, each material point only transmits linear momentum.

In reality the strain is not only due to stretch but also to the rotation of magnetic moments $\boldsymbol{m}_{i}$, which should be considered in some situations.

\section{Debye relaxation}

Magnetic viscosity - mathematically described by the parameter $\tau_{M}$, see [33] — causes a frequency-dependent behaviour in permeability and piezomagnetic material properties; the second is due to the coupling between magnetic and mechanical fields. From a mathematical point of view and in a first and good approximation, this dependence is represented by the Debye model that incorporates a parameter $f_{D}$, the simplest case of the Cole-Cole equation [34]:

$f_{D}(\omega)=\frac{1}{1+\left(\omega \tau_{M}\right)^{2}}$,

where $\omega$ denotes angular frequency. 
According to [35] the Debye relaxation is described by the spin-lattice model: the magnetic dipoles (spin) must reach thermodynamic equilibrium with its surroundings (lattice). Then, frequency-dependent material tensors are approximately split into static $(\cdot)_{s t}$ and dynamic $(\cdot)_{d y}$ contributions:

$$
\begin{aligned}
& \boldsymbol{\mu}(\omega) \approx \boldsymbol{\mu}_{s t} f_{D}+\boldsymbol{\mu}_{d y}\left(1-f_{D}\right) \\
& \boldsymbol{e}^{\varphi}(\omega) \approx \boldsymbol{e}_{s t}^{\varphi} f_{D}+\boldsymbol{e}_{d y}^{\varphi}\left(1-f_{D}\right)
\end{aligned}
$$

Combining (45) and (44), two limit cases are obtained:

$\triangleright\left(\omega \tau_{M}\right)^{2}<<1 \Rightarrow \boldsymbol{\mu}, \boldsymbol{e}^{\varphi} \rightarrow \boldsymbol{\mu}_{s t}, \boldsymbol{e}_{s t}^{\varphi}$ : the spin-lattice system are in isothermal equilibrium state and, consequently, no dissipation appears.

$\triangleright\left(\omega \tau_{M}\right)^{2}>>1 \Rightarrow \boldsymbol{\mu}, \boldsymbol{e}^{\varphi} \rightarrow \boldsymbol{\mu}_{d y}, \boldsymbol{e}_{d y}^{\varphi}$ : the temperature of the spin increases while that of the lattice does not change; then dissipation is present.

From a numerical point of view and in order to study the influence of the Debye relaxation in the response of magnetostrictive materials, case III from the last section is studied again. Values $\tau_{M}=0,17,23(\mathrm{~ms})$ are considered: the zero corresponds to the basic case and the other two are empirically adjusted in [35] for the similar magnetostrictive material PMN-PT/CFO. Also, two time-dependent electric currents of low-medium frequency similar to those presented in [8] (see sketches in Fig. 8 right column) are prescribed:

1) Sinusoidal $I_{a}=15 \sin (400 \pi t)$,

2) Harmonic $I_{a}=15[\sin (300 \pi t)+\sin (100 \pi t)]$,

Figure 8 plots the FE results for the two currents and for each of the three relaxation times: $\boldsymbol{M}$ from (2) versus $\boldsymbol{H}$ (left column), and $\boldsymbol{S}$ versus $\boldsymbol{M}$ (right column), all of them along the Terfenol-D rod length described before.

Several points on the results can be highlighted:

a) No hysteretic responses appear when $\omega \tau_{M}=0$ and, consequently, there is not dissipation.

b) The left-column figures show a linear response (with loops) since the magnetic constitutive (25) bottom is linear. On the contrary, in the right-column figures the magneto-elastic coupling (25) top exhibit clear non-linear responses due to the MST.

c) As expected, the loop width, or dissipation, increases with $\tau_{M}$

d) In the left column, the average slope of the loop slightly decreases with the increase of $\omega \tau_{M}$, or the decrease of $f_{D}$ in (44), since the ratio between $\boldsymbol{\mu}_{s t}$ and $\boldsymbol{\mu}_{d y}$ is altered.

In the last item, one has to consider that the total $\boldsymbol{\mu}$ of (45) top is the slope of the curves $\boldsymbol{B}$ vs. $\boldsymbol{H}$.
In the bottom row, several loops of different width appear since the current includes several amplitudes and frequencies.

\section{Concluding remarks}

This work has presented a three-dimensional, monolithic, non-linear, dynamic and dissipative finite element formulation to model giant magnetostrictive actuators.

Theoretically, the approach is consistent since the constitutive equations have been specially obtained (for this application) from a thermodynamic potential and the dissipations - time-dependent constitutive equationsfrom the entropy production, according to the nonequilibrium thermodynamics.

Numerically, one of the novelties is the combination of Newmark method for the time integration of the mechanical field and convolution integrals to integrate the time-dependent magnetic constitutive equation. The use of this convolution allows to solve part of the equation in closed form and, consequently, it is more accurate than other numerical schemes such as finite differences. The problem is non-linear due to the existence of the MST, quadratically dependent on the magnetic field; this problem has been solved by a monolithic finite element formulation based on residuals, minimized by the Newton-Raphson algorithm.

The limitations are: small strain and linear elasticity for the mechanic field; absence of free electric currents (magnetic scalar-based formulation); electromagnetic frequencies greater than mechanical one; and, absence of thermal effects.

In conclusion, the present formulation is more robust than empirical formulations based on hysteretic operators and it could be used to solve, among other objectives, inverse problems for a proper characterization of magnetostrictive materials. 

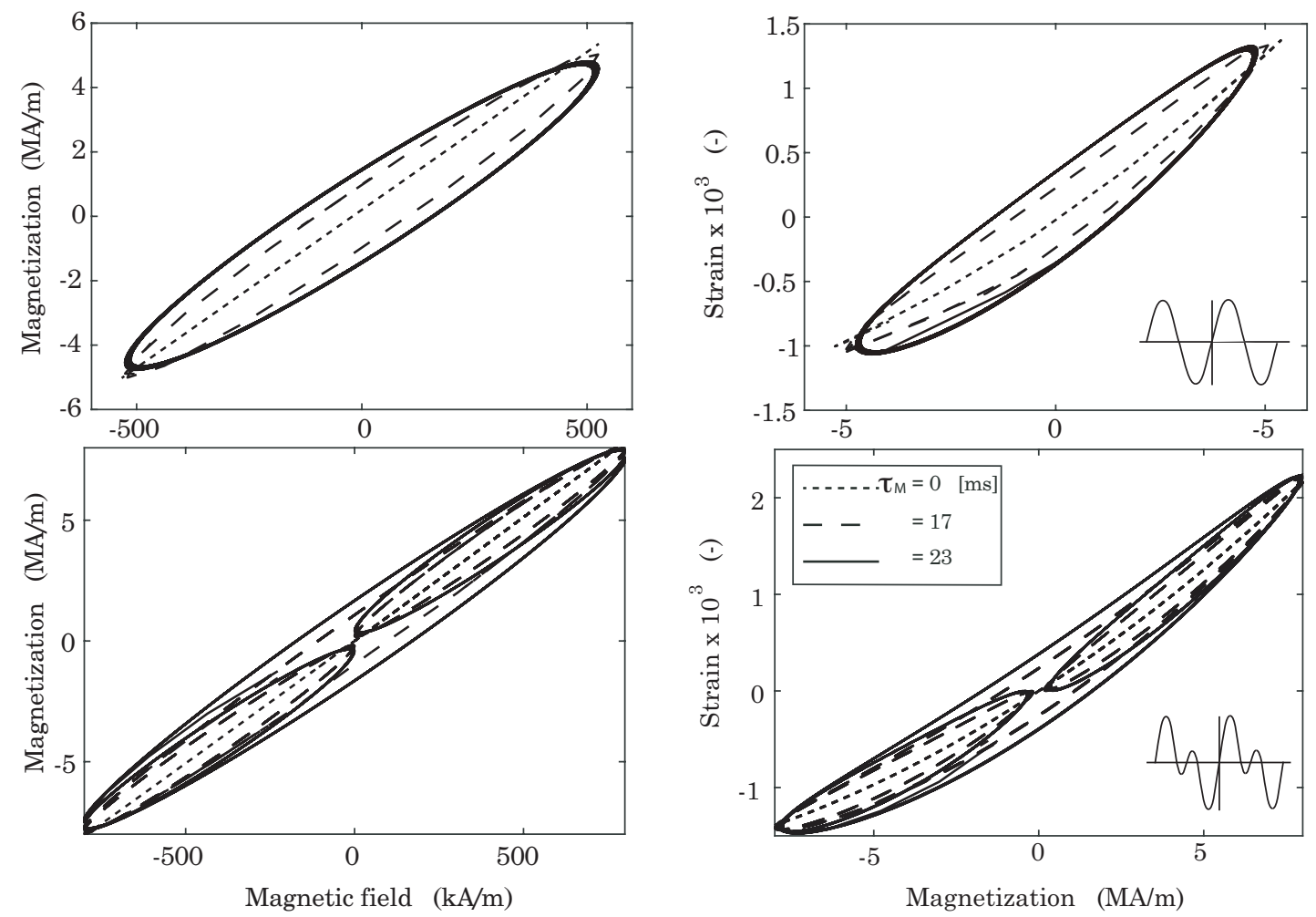

Fig. 8 Magnetization (left column) and strain (right column) along longitudinal axis from Fig. 5 vs. prescribed magnetic field for three relaxation times. Top: i) pure sinusoidal current; bottom: ii) harmonic current.

\section{Appendix}

For the FE implementation, the tangent stiffness $\mathcal{K}$ and mass $\mathcal{M}$ matrices may be explicitly expressed as:

$$
\begin{aligned}
\mathcal{K}_{a b}^{u u} & =\int_{\Omega_{e}} \mathcal{B}_{a}^{s y \top} \frac{\partial \boldsymbol{\sigma}_{T}^{n+1}}{\partial \tilde{\boldsymbol{u}}_{b}^{n+1}} \mathrm{~d} \Omega_{e}, \\
\mathcal{K}_{a b}^{u \varphi} & =\int_{\Omega_{e}} \mathcal{B}_{a}^{s y \top} \frac{\partial \boldsymbol{\sigma}_{T}^{n+1}}{\partial \tilde{\varphi}_{b}^{n+1}} \mathrm{~d} \Omega_{e}, \\
\mathcal{K}_{a b}^{\varphi u} & =-\int_{\Omega_{e}} \mathcal{B}_{a}^{\top} \frac{\partial \boldsymbol{B}^{n+1}}{\partial \tilde{\boldsymbol{u}}_{b}^{n+1}} \mathrm{~d} \Omega_{e}, \\
\mathcal{K}_{a b}^{\varphi \varphi} & =-\int_{\Omega_{e}} \mathcal{B}_{a}^{\top} \frac{\partial \boldsymbol{B}^{n+1}}{\partial \tilde{\varphi}_{b}^{n+1}} \mathrm{~d} \Omega_{e}, \\
\mathcal{M}_{a b}^{u u} & =\int_{\Omega_{e}} \mathcal{N}_{a} \rho_{m} \boldsymbol{I} \mathcal{N}_{b} \mathrm{~d} \Omega_{e} .
\end{aligned}
$$

First, the mechanic and magnetic derivatives are calculated using (32) and (5) along with (10):

$$
\begin{aligned}
\frac{\partial \boldsymbol{\sigma}_{T}^{n+1}}{\partial \tilde{\boldsymbol{u}}_{b}^{n+1}}= & \mathbf{C} \mathcal{B}_{b}^{s y}+\frac{\left.\partial \boldsymbol{\sigma}_{M x}^{s y}\right|^{n+1}}{\partial \tilde{\boldsymbol{u}}_{b}^{n+1}}, \\
\frac{\partial \boldsymbol{\sigma}_{T}^{n+1}}{\partial \tilde{\varphi}_{b}^{n+1}}= & \left(\boldsymbol{e}^{\varphi}\right)^{\top} \boldsymbol{B}_{b}+\frac{\left.\partial \boldsymbol{\sigma}_{M x}^{s y}\right|^{n+1}}{\partial \tilde{\varphi}_{b}^{n+1}}, \\
\frac{\left.\partial \boldsymbol{\sigma}_{M x}^{s y}\right|^{n+1}}{\partial \tilde{\boldsymbol{u}}_{b}^{n+1}}= & \frac{1}{2}\left[\frac{\partial \boldsymbol{B}_{n+1}}{\partial \tilde{\boldsymbol{u}}_{b}^{n+1}} \boldsymbol{H}_{n+1}^{\top}+\boldsymbol{H}_{n+1}\left(\frac{\partial \boldsymbol{B}_{n+1}}{\partial \tilde{\boldsymbol{u}}_{b}^{n+1}}\right)^{\top}\right. \\
& \left.-\frac{2}{\mu_{0}} \frac{\partial \boldsymbol{B}_{n+1}}{\partial \tilde{\boldsymbol{u}}_{b}^{n+1}} \boldsymbol{B}_{n+1}^{\top} \boldsymbol{I}\right],
\end{aligned}
$$

$$
\begin{aligned}
\frac{\left.\partial \boldsymbol{\sigma}_{M x}^{s y}\right|^{n+1}}{\partial \tilde{\varphi}_{b}^{n+1}}= & \frac{1}{2}\left[\frac{\partial \boldsymbol{B}^{n+1}}{\partial \tilde{\varphi}_{b}^{n+1}}\left(\boldsymbol{H}^{n+1}\right)^{\top}+\boldsymbol{H}^{n+1}\left(\frac{\partial \boldsymbol{B}^{n+1}}{\partial \tilde{\varphi}_{b}^{n+1}}\right)^{\top}\right. \\
& -\boldsymbol{B}^{n+1} \boldsymbol{\mathcal { B }}_{b}^{\top}-\boldsymbol{B}_{b}\left(\boldsymbol{B}^{n+1}\right)^{\top} \\
& \left.-\frac{2}{\mu_{0}} \frac{\partial \boldsymbol{B}_{n+1}}{\partial \tilde{\varphi}_{b}^{n+1}} \boldsymbol{B}_{n+1}^{\top} \boldsymbol{I}\right] .
\end{aligned}
$$


Second, the time-dependent derivatives for the magnetic induction are obtained from (39) to give:

$$
\begin{aligned}
& \frac{\partial \boldsymbol{B}^{n+1}}{\partial \tilde{\boldsymbol{u}}_{b}^{n+1}}=[1-\phi(\Delta \tau)] \boldsymbol{e}^{\varphi} \mathcal{B}_{b}^{s y} \\
& \frac{\partial \boldsymbol{B}^{n+1}}{\partial \tilde{\varphi}_{b}^{n+1}}=-[1-\phi(\Delta \tau)] \boldsymbol{\mu} \mathcal{B}_{b} .
\end{aligned}
$$

Finally, this numerical formulation is implemented in the FE research software FEAP [20].

\section{References}

1. R. R. Orszulik and U. Gabbert, An interface between Abaqus and Simulink for high-fidelity simulations of smart structures, IEEE/ASME Transactions on Mechatronics, 21(2), 879-887, (2015)

2. J. Melchor and G. Rus, Torsional ultrasonic transducer computational design optimization, Ultrasonics, 54(7), 1950-1962, (2014)

3. F. U. Khan and I. Ahmad, Review of energy harvesters utilizing bridge vibrations, Shock and Vibration, 2016, 1$21,(2016)$

4. A. Melingui, O. Lakhal, B. Daachi, J. Bosco and R. Merzouki, Adaptive neural network control of a compact bionic handling arm, IEEE/ASME Transactions on Mechatronics, 20(6), 2862-2875, (2015)

5. M. Anjanappa and J. Bi, A theoretical and experimental study of magnetostrictive mini-actuators, Smart Materials \& Structures, 3, 83-91, (1994)

6. M. Anjanappa and J. Bi, Magnetostrictive mini actuators for smart structure applications, Smart Materials \& Structures, 3, 383-390, (1994)

7. R. Venkataraman, J. Rameau and P. S. Krishnaprasad, Characterization of an ETREMA MP 50/6 Magnetostrictive actuator, TR 98-1, Technical report of the Institute for System Research, University of Maryland at College Park

8. Z. Li, X. Zhang, G. Y. Gu, X. Chen and C. Y. Su, A comprehensive dynamic model for magnetostrictive actuators considering different input frequencies with mechanical loads, IEEE Transactions on Industrial Informatics, 12(3), 980-990, (2016)

9. J. L. Pérez-Aparicio and H. Sosa, A continuum threedimensional, fully coupled, dynamic, non-linear finite element formulation for magnetostrictive materials, Smart Materials and Structures, 13, 493-502, 2004

10. J. L. Perez-Aparicio, R. Palma and R. L. Taylor, Multiphysics and thermodynamic formulations for equilibrium and non-equilibrium interactions: non-linear finite elements applied to multi-coupled active materials, Archives of Computational Methods in Engineering, 23(3), 535-583, (2016)

11. K. S. Kannan and A. Dasgupta, A non-linear Galerkin finite-element theory for modeling magnetostrictive smart structures, Smart Materials and Structures, 6, 341-350, (1997)

12. J. Kiang and L. Tong, Nonlinear magneto-mechanical finite element analysis of Ni-Mn-Ga single crystals, Smart Materials and Structures, 19, 1-17, (2010)

13. P. Zhou, On the coupling effects between elastic and electromagnetic fields from the perspective of conservation of energy, Arxiv.org, arxiv.org/pdf/1512.04487.pdf, (2015)
14. C. Natale, F. Velardi and C. Visone, Identification and compensation of Preisach hysteresis models for magnetostrictive actuators, Phys. B Condens. Matter, 306(1), 161-165, (2001)

15. M. Kaltenbacher, M. Meiler and M. Ertl, Physical modeling and numerical computation of magnetostriction, International Journal for Computation and Mathematics in Electrical and Electronic Engineering, 28(4), 819-832, (2009)

16. K. Linnemann, S. Klinkel and W. Wagner, A constitutive model for magnetostrictive and piezoelectric materials, International Journal of Solids and Structures, 46, 1149-1166, (2009)

17. S. R. de Groot and P. Mazur, Non-equilibrium Thermodynamics, Dover, (1984)

18. J. L. Pérez-Aparicio, R. Palma, Pablo Moreno-Navarro, Elasto-thermoelectric non-linear, fully coupled, and dynamic finite element analysis of pulsed thermoelectrics, Applied Thermal Engineering, 107, 398-409, (2016)

19. R. Palma, J. L. Pérez-Aparicio and R. L. Taylor, Dissipative finite element formulation applied to piezoelectric materials with Debye memory, IEEE/ASME Transactions on Mechatronics, 23(2), 856-863 (2017)

20. R. L. Taylor, FEAP A Finite Element Analysis Program: User Manual, University of California, Berkeley, (2010)

21. G. Sardanashvily, Noether's Theorems. Applications in Mechanics and Field Theory, Springer-Verlag, (2016)

22. J. L. Jiménez, I. Campos and M. A. López-Mariño, Maxwell's equations in material media, momentum balance equations and force densities associated with them, The European Physical Journal Plus, 128(46), 1-6, (2013)

23. R. Palma, J. L. Pérez-Aparicio and R. L. Taylor, On the non-symmetry of the Maxwell stress tensor: A generalized continuum mechanics approach, International Journal of Engineering Science, submitted, (2019)

24. L. D. Landau and E. M. Lifshitz, Electrodynamics of Continuous Media, Pergamon Press Ltd, (1984)

25. H. J. Juretschke, Simple derivation of the Maxwell stress tensor and electrostictive effects in crystals, American Journal of Physics, 45(3), 277-280, (1977)

26. J. R. Reitz, F. J. Milford and R. W. Christy, Foundations of Electromagnetic Theory, Addison-Wesley Publishing Company, Inc, (1960)

27. H. B. Callen, Thermodynamics and an Introduction to Thermostatistics, John Wiley and Sons, Inc, (1985)

28. R. M. McMeeking and C. M. Landis, Electrostatic forces and stored energy for deformable dielectric materials, Journal of Applied Mechanics, 72, 581-590, (2005)

29. O. C. Zienkiewicz, R. L. Taylor and J. Z. Zhu, The Finite Element Method: Its basis and Fundamentals, 7th ed, Elsevier Butterworth-Heinemann, (2013)

30. G. Engdahl, Handbook of Giant Magnetostrictive Materials, Academic Press, (2000)

31. M. J. Dapino, A. B. Flatau and F. T. Calkins, Statistical analysis of Terfenol-D material properties, Journal of Intelligent Material Systems and Structures, 17(7), 587-599, (2006)

32. M. B. Moffett, A. E. Clark, M. Wun-Fogle, J. Linberg, J. P. Teter, and E. A. McLaughlin, Characterization of Terfenol-D for magnetostrictive transducers, The Journal of the Acoustical Society of America, 89(3), 1448-1455, (1991)

33. R. V. Telesnin and A. G. Shishkov, Effect of magnetic viscosity on the frequency properties of ferrites. Sovietic Physics JETP, 6(33), 649-652, (1958)

34. K. S. Cole and R. H. Cole, Dispersion and absorption in dielectrics - I alternating current characteristics. Journal of Chemistry Physics, 9(4), 341-352, (1941) 
35. A. J. Gualdi, M. L. Zabotto, D. Garcia, A. Bhalla, R. Gu, P. C. de Camargo and A. J. de Oliveira, Understanding the dynamic magnetization process for the magnetoelectric effect in multiferroic composites. Journal of Applied Physics, 119(12), 4110 (2016) 